\title{
Effect of Temperature Variation on Adhesive Contact Force Behavior and Deformation of Substrate Based on Atomic Scale
}

\author{
Jing-jing Chen ${ }^{{ }^{*}}$, Hui-cun Shen ${ }^{1}$, Hong-jun $\mathrm{Hu}^{1}$ \\ ${ }^{1)}$ School of Information \& Electrical Engineering, Ningde Normal University, Ningde 350108, China \\ "Corresponding author: chenjingjingfzu@126.com
}

Keywords: adhesive contact; temperature variation; the friction force; substrate deformation; molecular dynamics

\begin{abstract}
Molecular dynamics method is performed to investigate the force behavior between semi-spherical indenter and single crystal substrate based on eam and morse potentials and verlet algorithm. Effects of different temperature on the contact force, friction force, and normal force are compared, the resistance of substrate material and dislocation movement are analyzed. It shows that the single crystal copper material's resist deformation level is limited by low temperature, and more failure atoms were accumulated under the direction of indenter indenting in high temperature. More larger chip atoms volume were produced in front of indenter as sliding speed increases, and also the chip volume add with sliding distance increases. Furthermore, the dislocated band in basis lies at the angle of 45 degree with the movement direction of indenter in contact progress. This results have significance in improving contact performance and reducing the adhesive contact failure happens
\end{abstract}

\section{Introduction}

The adhesive contact force behavior at two surface contacts has long been considered as an important subject since the force behavior properties of a contact models can be strongly influenced by the deformation of the substrate, especially in different conditions (temperature, velocity, dimensions). However, the failure often caused by the friction and wear force of two surfaces in MEMS actuators[1]. The atomic details at the contact surface during sliding and indenting process had not been fully understand due to the limitations in experimental techniques despite its scientific importance from two aspects on the below. On the one hand, it may not be appropriate to apply the conventional theory to analyze the nano-scale adhesive contact and research the contact force behavior under different conditions. On the other hand, the experimental investigation on material nano-scale is constrained by the high costs, long time and large uncertainty of measurement and experiment conditions. Therefore, molecular dynamics method (MD) has become an useful tool and wildly applied to investigate the tribological behaviors and also observe the deformation of substrate on the conditions of temperature variation based on atomic scale.

Some researches adopt MD method to research the contact behaviors during in cutting or scratching or sliding process[2-5]. Q.X.Pei et al.[3] illuminated that the crystal orientation and cutting direction have a strong effect on material deformation, dislocation movement and cutting forces. Li et al.[6]suggested that the elastic deformation of surface layers is the main cause for the atomic scale stick-slip and the degree of commensurate atomic fit at the sliding interface had little influence on the stick-slip friction. Jun jie Zhang[7] shows that dislocations move well-regulated for lower velocities $(1$ and $10 \mathrm{~m} / \mathrm{s})$ than that for larger one $(100 \mathrm{~m} / \mathrm{s})$.Yan et al.[8] studied that the feed has a significant effect on the deformation of the machined surface, the scratching depth, scratching forces and potential energy. Konmanduri et al.[9] study showed that the friction coefficient was constant and independent of scratch depth at extremely low depths $(0.1-0.8 \mathrm{~nm})$. Mulliah et al. [10]studied the nano-scratch process of silver $\left(\begin{array}{lll}1 & 0 & 0\end{array}\right)$ by MD method. The results showed that the coefficient of friction is dependent on both the orientation of the indenter and the indentation depth, and the coefficient of friction increases as the depth increases. Temperature has its effects on surface 
roughness and volume of chips [11,12]. Lower temperature results in a smooth surface and smaller volume of chips.

All above literatures only consider the single process of scratching or sliding, Ignoring the actual process that tip indent substrate with a certain distance before start sliding along $\mathrm{x}$ direction. Kun Sun et al.[13] would not find the reason of the differences between contact force from MD computers and mached Hertz theory. In this work, three-dimensional MD method simulations based on EAM and Morse potentials and Verlet algorithm are applied to investigate the effects of system temperature $(20 \mathrm{~K}, 298 \mathrm{~K}, 500 \mathrm{~K})$ on contact force, friction force, normal force and friction coefficient during contacting and sliding process. At the same time, analyzed the features that chip volume produced and substrate deformation. Finally, according to the results of the comparisons, some suggestions on selecting temperature and sliding speed parameters for directing the relevant surface designs in MEMS/NEMS are given.

\section{Simulation methods}

The 3D adhesive contact modes in this study are a diamond indenter and a single crystal copper substrate, as illustrated in Fig.1. The size of substrate is $50 \mathrm{a} \times 37 \mathrm{a} \times 30 \mathrm{a}(\mathrm{a}: 0.36149 \mathrm{~nm})$. The single crystal copper substrate is consisted by 165000 atoms, the three orientations of substrate are in $x[100], y[010]$ and $z[001]$ in a face-centered cubic (FCC) copper lattice. A hemispherical diamond indenter with a radius of $2.5 \mathrm{~nm}$, consisting of 6522 carbon atoms, is used for the simulations. The indentation/slip plane is (001) and the sliding direction (x positive direction) is [100]. The letter of $\mathrm{d}$ in Fig.1(b) means the indentation depth, the indentation depth is $1 \mathrm{~nm}$ and the sliding distance is $4 \mathrm{~nm}$.

There are two stages in simulation: one is the indenting process, and the other is sliding process. The substrate contains three layers of atoms, namely, boundary atoms, thermostat atoms and Newtonian atoms in Fig.1(b). The motions of thermostat atoms and Newtonian atoms obey classical Newton's second law, which are integrated by verlet algorithm[14] with time-step1fs. Periodic boundary conditions are imposed in the $\mathrm{x}$ and $\mathrm{z}$ direction for all simulations. There are three different atomic interactions in MD simulation: the $\mathrm{C}-\mathrm{C}$ atoms interaction in the diamond indenter; the $\mathrm{Cu}-\mathrm{Cu}$ atoms interaction in the substrate; and the $\mathrm{Cu}-\mathrm{C}$ atoms interaction between the substrate and the indenter. The EAM(embedded atomic method) potentials, contributed by Mishin Y[15], is adopted to describe the $\mathrm{Cu}-\mathrm{Cu}$ atoms interaction, the $\mathrm{C}-\mathrm{C}$ atoms interactions in the diamond indenter are ignored due to the diamond indenter as a rigid body, the parameters of $\mathrm{Cu}-\mathrm{C}$ atoms interaction are adopted by the Morse potentials[16]. The temperature and total energy in system are relaxed through the NVT ensemble for 8ps, and then through the NVE ensemble for another 8ps in MD simulations, so that the temperature and total energy in system to be reach thermodynamics balance. The indenter is moved at contact speed $100 \mathrm{~m} / \mathrm{s}$ along the y negative direction at firstly, and then sliding the substrate along the x positive direction. The boundary atoms are held fixed to prevent the system from translating during the processes. Heat dissipation is carried out through keeping the thermostat atoms at a constant temperature of $298 \mathrm{~K}$ by Berendsen thermostat, which rescales their velocities every timestep $1 \mathrm{fs}$. 

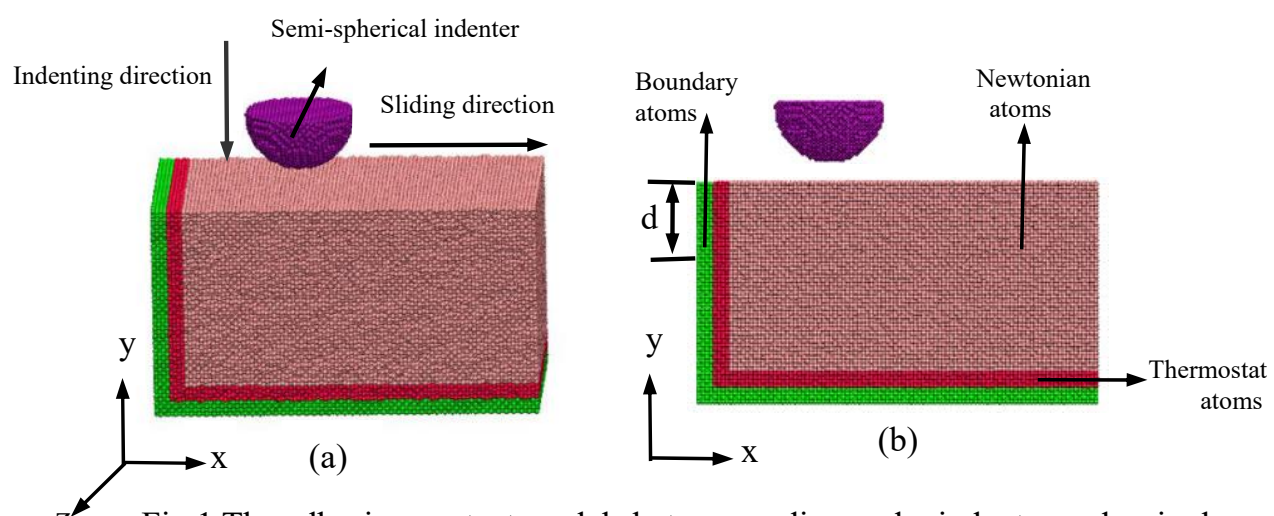

Fig. 1 The adhesive contact models between a diamond indenter and a single crystal $\mathrm{Cu}$ substrate. (a): three-dimensional model (b): two-dimensional model

\section{Results and analysis}

\subsection{Contact process}

Elastic response can be characterized by Hertz theory [17] during the indenting contact process, which is described as follows:

$$
\begin{gathered}
F=\frac{4}{3} E_{1}^{*} R^{1 / 2} \delta^{3 / 2} \\
E^{*}=\left(\frac{1-v_{s}^{2}}{E_{s}}-\frac{1-v_{i}^{2}}{E_{i}}\right)^{-1} \ldots \ldots \ldots \ldots \ldots \ldots \ldots \ldots \ldots \ldots \ldots \ldots \ldots \ldots \ldots \ldots \ldots \ldots \ldots
\end{gathered}
$$

Where $\mathrm{F}$ is the applied load, $\mathrm{R}$ is indenter radius, $\mathrm{E}$ is the reduced modulus, $\delta$ is the contact displacement. $E_{s}$ and $v_{s}, E_{i}$ and $v_{i}$ are the Young's modulus, Poisson's ratio of the substrate and the indenter. $E_{i}=1050 \mathrm{GPa} 、 E_{s}=115 \mathrm{GPa} ; \quad v_{i}=0.1 、 v_{s}=0.25$, the composite modulus of elasticity $E^{*}=138.9 \mathrm{GPa}$ is obtained through above equation(2). Point $\mathrm{A}$ (Fig.2) indicates the begin contact of Hertz theory. Point B(Fig.2)indicates contact force got to $133.5 \mathrm{nN}$ when indentation dept reached $\delta=\mathrm{d}=0.603 \mathrm{~nm}$. this computer parameters are adopted into equation (3) and obtained composite modulus of elasticity $E_{1}^{*}=135.3 \mathrm{GPa}$. The error value between $E^{*}$ and $E_{1}^{*}$ is strictly controlled within $2.5 \%$. Therefore, adhesive contact model and parametres select are constructed by MD methods is credible.

As shown in Fig.2, the contact force values between MD computer and Hertz theory matching are gradually raising as the increasing of indenting distance. Firstly, the contact force measured is larger than matched by the Hertz theory before indent depth $d<0.603 \mathrm{~nm}$. The main reason belongs to the effects of substrate elastic deformation in Hertz theory, but disregards the existence of adhesive and surface force between contact surface. Secondly, when indent depth $d>0.603 \mathrm{~nm}$, the contact force measured is smaller than matched Hertz theory have two main factors. 


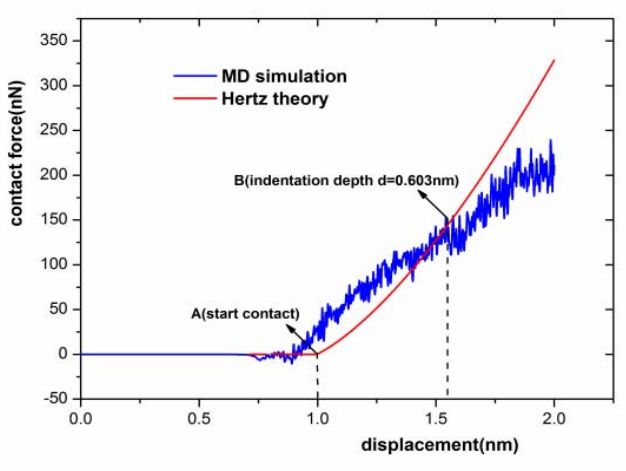

Fig. 2 the cures of the contact force vs displacement between MD simulation and Hertz theory

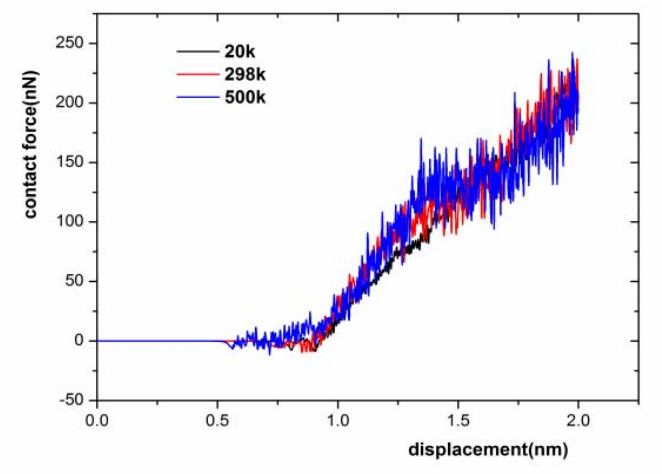

Fig. 3 the cures of the contact force vs displacement at different temperature $(20 \mathrm{k} \sim 500 \mathrm{k})$

One the one hand, due to it overlooks the influence on plastic deformation of substrate in Hertz theory. On the other hand, substrate happened transition from elastic deformation to plastic deformation. From Fig.3, we can find out that temperature has little effects on contact force. The higher system temperature is, the larger the oscillating amplitude of curve, which is about the contact force changing with displacement will be. This is because the higher the temperature is, the more frequently the atoms of basis move(we can see from Fig.4(a c) ) and the more unstable of applied force of atoms between $\mathrm{Cu}-\mathrm{Cu}$ and $\mathrm{Cu}-\mathrm{C}$.

The center symmetry parameter (CSP) value in Fig.4 is used to identify dislocations and other lattice defects[18]. The CSP values is zero for an atom in a perfect face centered cubic lattice and positive value for an atom which is part of a defect such as a dislocation or a surface. The effects of different temperature $(20 \mathrm{k} \sim 500 \mathrm{k})$ towards substrate can be figured out from figure 4 . Because the elevated temperature softens the copper material, the deformation level of substrate increases with the temperature, so that the substrate more prone to transform. Under the same thermal circumstance, because the contact force increases with rising of displacement of indenter and temperature of local area, the deformation of basis is more serious with the aggrandizement of indenting distance. Compared with figure $4(\mathrm{a} \sim \mathrm{c})$, we find that if the temperature is increasing, the blue failure atoms of basis manifolds. This indicates that the movement of atoms in the basis is more intense for the elevated temperature. When the temperature is identical, the increased pushing distance will result in more worn blue atoms in basis. This is because the raised pushing distance brings about the rapidly increased temperature of local contact area. Furthermore, the dislocated atoms continuously accumulate under the indenter and grow in quantity with the augment of temperature and pushing distance. In addition, the dislocated band on the both sides of basis lies at the angle of 45 degree with the trajectory of indenter. 
(a) $\mathrm{T}=20 \mathrm{k}$

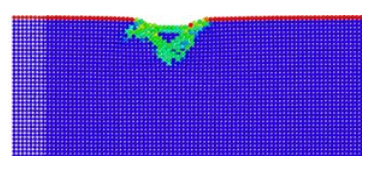

$\mathrm{d}=0.4 \mathrm{~nm}$

(b)

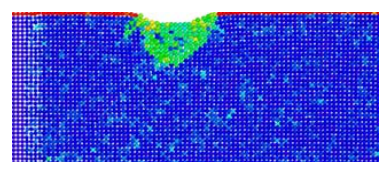

$\mathrm{d}=0.4 \mathrm{~nm}$

(c)

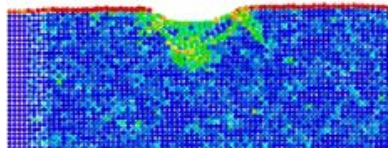

$\mathrm{d}=0.4 \mathrm{~nm}$

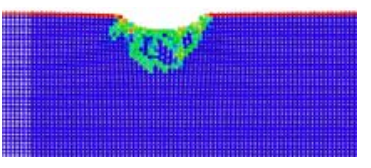

- in
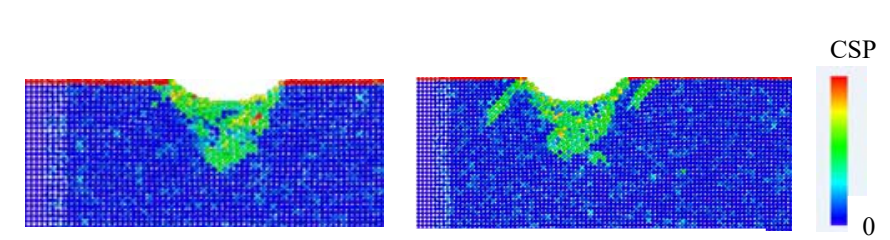

$\mathrm{d}=0.7 \mathrm{~nm}$

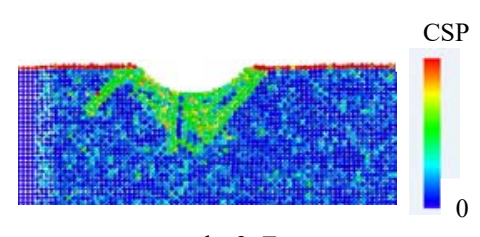

$\mathrm{d}=0.7 \mathrm{~nm}$

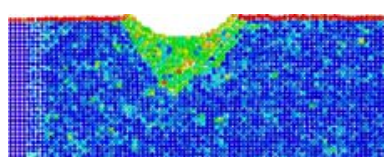

Fig. 4 The substrate deformation during the indenting contact process under different temperature

\subsection{Sliding process}

It is seen that grinded atoms' volume on the surface of substrate thickens with sliding distance increasing in Fig.5. The reason is that, during the slipping process, stress concentration exists on contact area between indenter and substrate, and majority of heats comes from the extrusion and friction between them, results in plastic deformation of atoms among local contact area lastly, due to the extruding, frictional and shearing force.

Cuttings leave behind along the moving direction of indenter, in addition, nearly all of the chemical and kinetic energy are transformed into thermal energy. Even though most of the energy are carried off by cuttings without energy exchange with the outside system so that the temperature of substrate risegradually as sliding distance increase (in Fig.5). The elevated temperature will soften the surface material of substrate and lead to a more fragile substrate surface and more chips volume of cuttings. As seen from Fig.8, it indicates that grinding atoms continuously accumulate in front of indenter along indenter sliding direction $(\mathrm{x})$. Furthermore, with the augment of system temperature increase, the higher temperature is, the less time dislocated atoms apart from contact area, and the easyier accumulated dislocation atoms are soften, so that material of contact surface happened adhesive contact failure. Tab.1 also shows that friction coefficient decreases with the augment of system temperature increases. friction coefficient $(\mu)$, a ratio of the average frictional force to the average normal force, can be used to manifest the extent of damage-resist level with three kinds of temperature. Then, it can be known that the resistive damage level of copper substrate material is lower when its temperature are higher in sliding process. Furchermore, we can also know about the friction force and normal force are decreases with system temperature increases in Tab.1. Therefore, during processing and manufacturing of MEMS surface material, in order to reduce contact surface deformation and damage, and to make the material surface work fine and to improve the contact performance. It is essential to consider the effect of temperature variation on adhesive contact behavior. 
(a) $\mathrm{T}=20 \mathrm{k}$

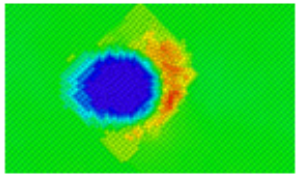

$\mathrm{s}=1.4 \mathrm{~nm}$

(b) $\mathrm{T}=298 \mathrm{k}$

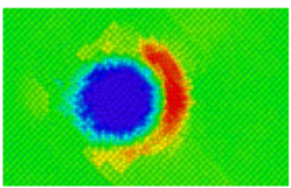

$\mathrm{s}=1.4 \mathrm{~nm}$

(c) $\mathrm{T}=500 \mathrm{k}$

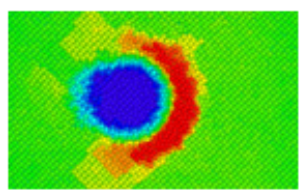

$\mathrm{s}=1.4 \mathrm{~nm}$

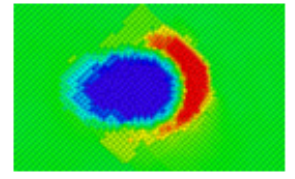

$\mathrm{s}=2.4 \mathrm{~nm}$

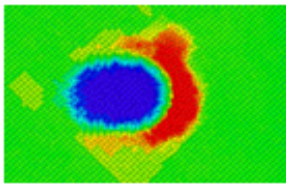

$\mathrm{s}=2.4 \mathrm{~nm}$

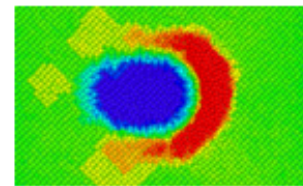

$\mathrm{s}=2.4 \mathrm{~nm}$

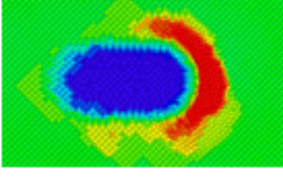

$\mathrm{s}=4 \mathrm{~nm}$

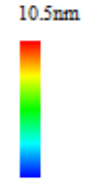

$9 \mathrm{~nm}$

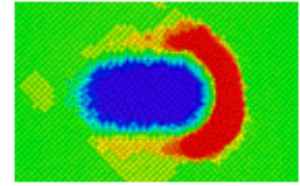

$\mathrm{s}=4 \mathrm{~nm}$

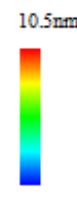

$9 \mathrm{~nm}$

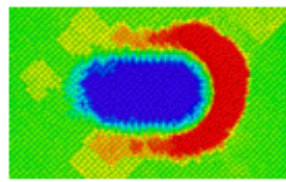

$10.5 \mathrm{~nm}$

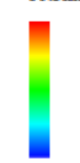

$9 \mathrm{~nm}$

Fig.5 the effect of substrate deformation during different sliding speed $(100 \mathrm{~m} / \mathrm{s} \sim 300 \mathrm{~m} / \mathrm{s})$ based on $298 \mathrm{k}$

Table 1 the effects of temperature on average force and normal force and friction coefficient with sliding speed $100 \mathrm{~m} / \mathrm{s}$

\begin{tabular}{|c|c|c|c|}
\hline $\begin{array}{c}\text { emperature } \\
(\mathrm{k})\end{array}$ & $\begin{array}{c}\text { Average friction } \\
\text { force }(\mathrm{nN})\end{array}$ & $\begin{array}{c}\text { Average normal } \\
\text { force(nN) }\end{array}$ & $\begin{array}{c}\text { Friction coefficient } \\
\mu\end{array}$ \\
\hline $20 \mathrm{k}$ & 86.389 & 85.067 & 1.01 \\
\hline $298 \mathrm{k}$ & 79.675 & 88.258 & 0.902 \\
\hline $500 \mathrm{k}$ & 68.447 & 79.545 & 0.86 \\
\hline
\end{tabular}

\section{Conclusion:}

In this article, we adopt MD methods to simulate the indenting and slipping process between the indenter and the single crystal copper substrate and find out that system temperature have a great influence on contact force, friction force, normal force and resist deformation level of substrate.

(1). The higher the system temperature is, the more frequently the atoms in the substrate will move, the higher the vibration amplitude of the curve that is about the relation of contact force and the displacement indenter will be and the larger the plastic deformation of substrate will become.

(2). The rising slipping temperature contributes to engender abrasive dusts from the substrate surface, and the higher the system temperature is, the more abrasive atoms will accumulate in front of indenter. In addition, the friction coefficient decreases with the rising of system temperature. This suggests that the single crystal copper material' s resist deformation level is limited.

\section{Acknowledgments}

This project is supported by Fujian province Science Foundation (2017J01709), and also provided by Ningde normal university as well as education department of Fujian province under grant No: (2015Q09, 2015Q10) and (JAT160540, JAT160535).

\section{References}

[1] D.M. Tanner. in: Proceedings of the 22nd International Conference in Microelectronics, NIS, 
Yugoslavia, May 2000, pp. 97-104.

[2] M.H.Cho, S.J.Kim, D.-S.Lim, H.Janga, Atomic scale stick-slip caused by dislocation nucleation and propagation during scratching of a $\mathrm{Cu}$ substrate with a nano-indenter: a molecular dynamics simulation, Wear 259 (2005) 1392-1399.

[3] Q.X. Pei, C. Lu, H.P. Lee, Large scale molecular dynamics study of nano-metric machining of copper, Computational Materials Science 41 (2007) 177-185.

[4] B. Shen, F. Sun, Molecular dynamics investigation on the atomic scale in dentation and friction behaviors between diamond tips and copper substrate,Diamond\& Related Materials, 19(2010): 723-728.

[5] P. Zhu,Y. Hu,H.Wang,et al.,Study of effect of indenter shape in nano-metric scratching process using molecular dynamics,Materials Science Engineering A 528 (2011)4522-4527.

[6] B. Li, P.C. Clapp, J.A. Rifkin, X.M. Zhang, J. Appl. Phys. 90 (2001):3090-3094.

[7] Junjie Zhang, Tao Sun, Yongda Yan, Yingchun Liang, Molecular dynamics study of scratching velocity dependency in AFM-based nano-metric scratching process, Materials Science and Engineering A 505 (2009) 65-69.

[8] Y. Yan, T. Sun, S. Dong, Y. Liang, Study on effects of the feed on AFM-based nano-scratching process using MD simulation[J], Computational Materials Science 40(2007) 1-5.

[9] Komanduri, R.; Chandrasekaran, N.; Raff, L.M. MD simulation of nano-metric cutting of single crystal aluminum-effect of crystal orientation and direction of cutting. Wear 2000, 242, 60-88.

[10] [10] D. Mulliah, S.D. Kenny, R. Smith, C.F. Sanz-Navarro, Molecular dynamic simulations of nano-scratching of silver (1 0 0), Nanotechnology 15 (2004) 243-249.

[11] Y. Isono, T. Tanaka, JSME Int. J. A 40 (1997) 211-218.

[12] Y. Isono, T. Tanaka, JSME Int. J. A 42 (1999) 158-166.

[13] Kun Sun,Liang Fang, ZhiyangYan, Jiapeng Sun, Atomistic scale tribological behaviors in nano-grained and single crystal copper systems, Wear303(2013)191-201.

[14] Loup Verlet. Computer 'experiments' on classical fluids. I. Thermodynamical properties of Lennard-Jones molecules. Phys. Rev. 1967,159. 98-103.

[15] Mishin Y, Mehl M J, Papaconstantopoulos D A, Voter A F, Kress J D. Structural stability and lattice defects in copper: $\mathrm{Ab}$ initio, tight-binding, and embedded-atom calculations[J]. Physical Review B, 2001, 63 (2001) 224106.

[16] Morse P M. Diatomic molecules according to the ware mechanics, II. Vibrational levels. Phys Rev. 1929, 34. 57-64.

[17] K.J. Johnson, Contact Mechanics, Cambridge University Press, 1985.

[18] R.Smith, D.Christopher, S.D.Kenny. Defect Generations and Pile-up of Atoms during Nanoindentation of Fe Single Crystal. Phys Rev B, 67(2003): 245405. 